\title{
Prediction of Dynamic Response of Vibration Isolated Railway Obstacle Detection System
}

\author{
Milan Banić1, Ivan R. Pavlović ${ }^{\text {, Aleksandar Miltenović }}$, \\ Miloš Simonović ${ }^{1}$, Marko Mladenovićé, Dragan Jovanović1, \\ Milan Rackov ${ }^{3}$ \\ ${ }^{1}$ University of Niš, Faculty of Mechanical Engineering \\ Aleksandra Medvedeva 14, 18000 Niš, Serbia; milan.banic@masfak.ni.ac.rs, \\ pivan@masfak.ni.ac.rs, aleksandar.miltenovic@masfak.ni.ac.rs, \\ milos.simonovic@masfak.ni.ac.rs,dragan.jovanovic@masfak.ni.ac.rs \\ ${ }^{2}$ Harder Digital SOVA, Bulevar Cara Konstantina 80, 18000 Niš, Serbia; \\ marko.mladenovic@hdsova.rs \\ ${ }^{3}$ University of Novi Sad, Faculty of Technical Science \\ Trg Dositeja Obradovića 6, 21000 Novi Sad, Serbia; racmil@uns.ac.rs
}

Abstract: The prototype of the on-board Obstacle Detection System (ODS), for GoA2 freight trains, was developed in the frame of H2020 project SMART. The developed prototype uses vision-based sensors, for environmental perception and obstacle detection. As severe image distortions while capturing imaging data can occur due to vibrations of the moving vehicle, the ODS of autonomous rail vehicles must be isolated from vibrations transmitting from the vehicle to have reliable image acquisition and post process analysis. The passive vibration isolation system was specially designed for suppression of moving rail vehicle vibrations during development of the ODS prototype. The paper presents the designed passive vibration system, as well as, experimental verification of its performance in operational conditions. Furthermore, two viscoelastic constitutive models, Voigt-Kelvin and Voigt-Maxwell, were applied to predict the dynamic response of vibration isolated ODS by using the real experimental data as system excitation.

Keywords: dynamic response; railway obstacle detection; vibration isolation; transmissibility of vibrations

\section{Introduction}

In many applications, the presence of harmful vibrations is unavoidable which is reducing the designed performance of machines and systems while emitting noise to environment [1]. The harmful vibrations in engineering, not only affect the use and working efficiency of precision instruments, but they may also trigger damage 
and accordingly, compromise structural integrity [2] [3]. Cameras and other vision sensors, as precision instruments mounted on vehicles, are especially sensitive to vibrations [4].

Autonomous rail vehicles for environment perception and obstacle detection by vision-based sensors must have image stabilization to have reliable image acquisition and post process analysis [5]. It can be found in many sources that, at frequencies greater than $5 \mathrm{~Hz}$, severe image distortions can occur [6]. With high image resolution, large optical zooming of the image and at low lighting conditions, the image distortion problem, due to vibrations, becomes more notable [6]. Many different image stabilization techniques have been developed to prevent distortion of image quality due to vibrations. Image stabilization techniques can be classified into four major categories: optical stabilization, digital stabilization, electro-mechanical and mechanical stabilization [7].

Mechanical stabilization operation principle is the isolation of the vision-based sensors from the vibration source. This way, vibrations cannot be suppressed completely, but amplitude of vibrations are significantly reduced, enabling further optical or digital stabilization [8]. Mechanical stabilization is done by implementing isolation devices made from materials possessing properties of elasticity and damping [9]. The mechanical isolators for imaging applications can be categorized in three major groups: solid viscoelastic dampers (rubber or rubber - metal springs); hysteretic isolators (metal springs, wire rope isolators); hysteretic-viscoelastic fluid isolators (combination of metal springs with fluid dampers). Isolation devices can realize vibration isolation in a wide range of frequencies, but only when the excitation frequency is $\sqrt{2}$ times higher than the natural frequency of vibrating system [10]. The rubber or rubber-metal springs are most often used as mechanical stabilization vibration isolators. Special types of rubber-metal springs were developed for isolation of sensitive instrumentation, measuring equipment and vision-based sensors. Noted springs for sensitive equipment have low natural frequency and ability to isolate vibrations in all three directions - vertical, lateral, and longitudinal.

Prediction of dynamic response, transmissibility and stability are major concern during the design of rubber-metal vibration isolators; thus a large number of authors deals with noted topics. The authors use analytical models solved directly or numerically [11-14] and finite element simulations with rubber constitutive models [15-17] to predict dynamic response and the transmissibility of vibration isolators. In most of the studies by other authors, the disturbing force is modelled as a sinusoidal input or as a white noise [18] to predict the dynamic response, transmissibility, and stability.

The development of railway transportation will be an increasingly important task for further developing of society in the $21^{\text {st }}$ Century [19]. To contribute to railway transport increase of quality, effectiveness, and capacity while opposing the strong competence of other transport means [20], the prototype of the on-board Obstacle 
Detection System (ODS) for GoA2 freight trains was developed in the frame of H2020 project SMART [21]. As the ODS uses vision-based sensors for environment perception, the passive vibration isolation system was specially designed for suppression of vibrations transmitting from the moving vehicle to the ODS. The paper presents the designed passive vibration system, as well as experimental verification of its performance in operational conditions for a cargo train in service. Furthermore, the viscoelastic Voigt-Kelvin and Voigt-Maxwell constitutive models were applied to predict the dynamic response by using the real experimental data for excitation of the system. The parameters of Voigt-Kelvin and Voigt-Maxwell models were determined by Monte Carlo simulation.

\section{Vibration Isolation of the Railway ODS}

The on-board freight train ODS prototype integrates three RGB cameras, thermal, night vision camera, and a 3D laser scanner (LiDAR) integrated into custom developed housing [8] as shown in Figure 1. The ODS housing is supported by the mounting plate rigidly connected to locomotive car body below the locomotive headlights. To prevent transmission of vibrations from the moving vehicle, via the mounting plate, to the ODS housing and thus, vision-based sensors, a passive vibration isolation system was designed.

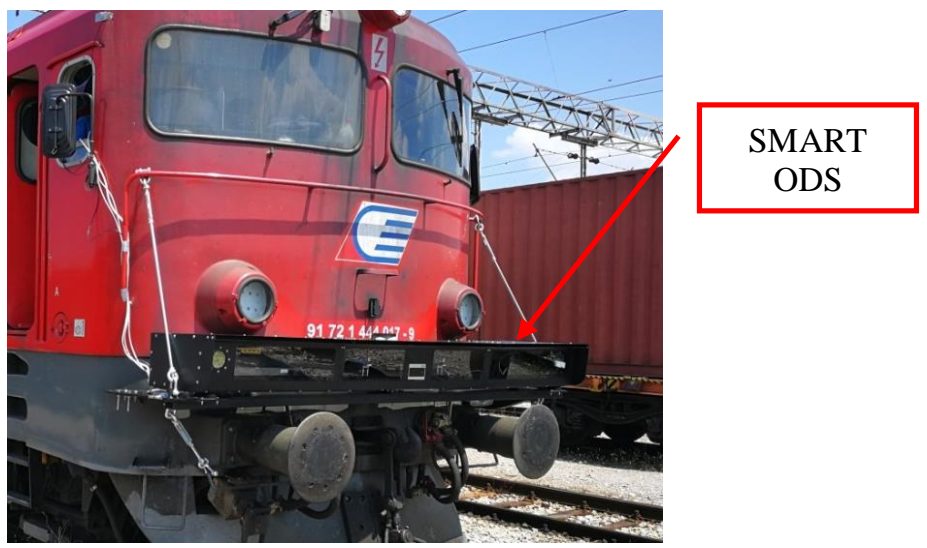

Figure 1

The SMART ODS prototype mounted onto the Serbia KARGO 444-018 locomotive [22]

As previously stated, for vibration isolation it is necessary that excitation frequency is $\sqrt{2}$ times higher than the natural frequency of vibrating system ODS housing and isolator assembly. The problem with vibrations transmitting from the rail vehicle to the ODS lies in the fact that there are multiple excitation frequencies which are not known. If one observes the diagram from Figure 2 it is 
evident that series 444 locomotive (SMART ODS evaluation vehicle) traveling at $75 \mathrm{~km} / \mathrm{h}$ at straight line has multiple resonant frequencies. In such case, widely adopted approach is to lower the vibrating system natural frequency, as much as possible, to achieve higher ratio between the excitation and vibrating system frequency. This is achieved by lowering the isolator stiffness which leads to larger displacement, thus lower vibrating system natural frequency, but the expense of static bearing capacity as it can become insufficient.

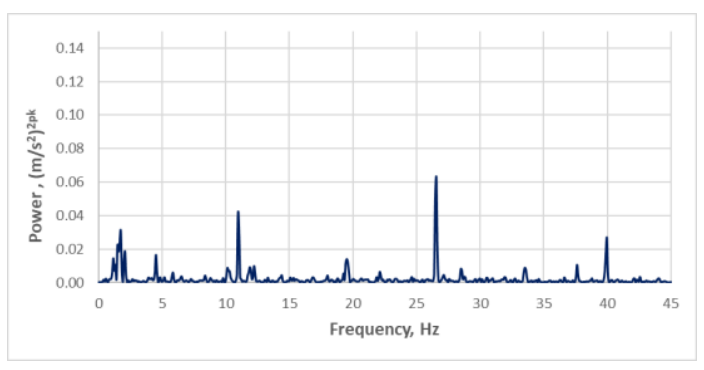

Figure 2

Vibration spectre of Serbia KARGO series 444 locomotive car body at $75 \mathrm{~km} / \mathrm{h}$ on straight line [7]

Furthermore, the damping ratio should be as large as possible as high damping will reduce the maximum possible transmissibility when the excitation frequency value is around natural frequency of the vibrating system. By analysis of vibration spectre given in Figure 2, it is evident that the natural frequency of the vibrating system must be below $8 \mathrm{~Hz}$ to isolate vibrations for a vehicle resonant frequency of around $11.5 \mathrm{~Hz}$. The first 2 resonant frequencies (at $\approx 2$ and $\approx 4 \mathrm{~Hz}$ ) can be neglected due to lower amplitude and the already stated fact that vibrations bellow $5 \mathrm{~Hz}$ will not downgrade quality of acquired images or can be easily mitigated with digital and optical stabilization. Based on requirements discussed above and due to good damping characteristics, and good static bearing capacity it was decided to design the vibration isolation system with rubber - metal springs. In principle, mechanical suppression of low frequency vibrations is possible only with a negative damping system which is not feasible for usage on the vehicle. The goal of adopted approach is to have a simple and low-cost vibration suppression system with proven service reliability. Furthermore, it was envisioned to use rubber-metal mounts already available on the market to decrease the system costs [23].
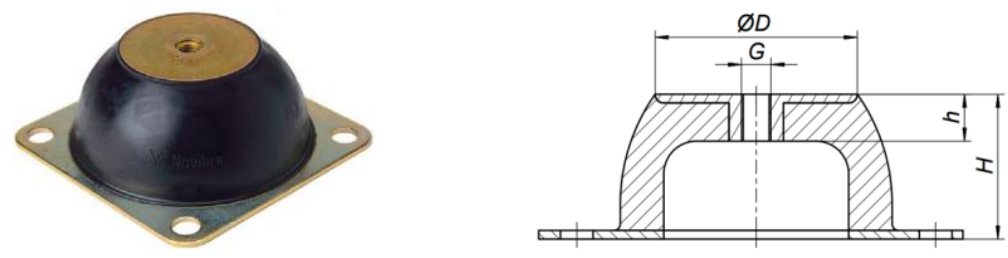

Figure 3

Bell-shaped rubber metal spring [24] 
As desired vibration system natural frequency must be below $8 \mathrm{~Hz}$, the selection was made to use the bell-shaped mount (Figure 3) which has a large deflection, as it is considered as most suitable rubber-metal mount for isolating the low frequency vibrations. Furthermore, it isolates vibration in all three directions, and it is suitable for shock suppression due to large deflection capacity.

The mass of the integrated ODS housing was intentionally increased to $160 \mathrm{~kg}$ by adding of ballast to increase the spring deflection and system inertia [25]. The number of mounts was selected as 4 to keep the uniform distribution of load across mounts and increase system stability. By usage of Trelleborg selection diagrams [24], the mount M50-40 was selected according to load per mount of 40 $\mathrm{kg}$. The selected mount provides vibrating system natural frequency of $7.5 \mathrm{~Hz}$, which is agreement with the requested value $<8 \mathrm{~Hz}$. The resonance region for selected mount is between 5.5 and $10.5 \mathrm{~Hz}$ which ensures that all the vehicles resonant frequencies are outside the vibrating system resonance region. For largest vehicle resonant frequency of $27 \mathrm{~Hz}$ (Figure 2), the isolation efficiency is $72 \%$.

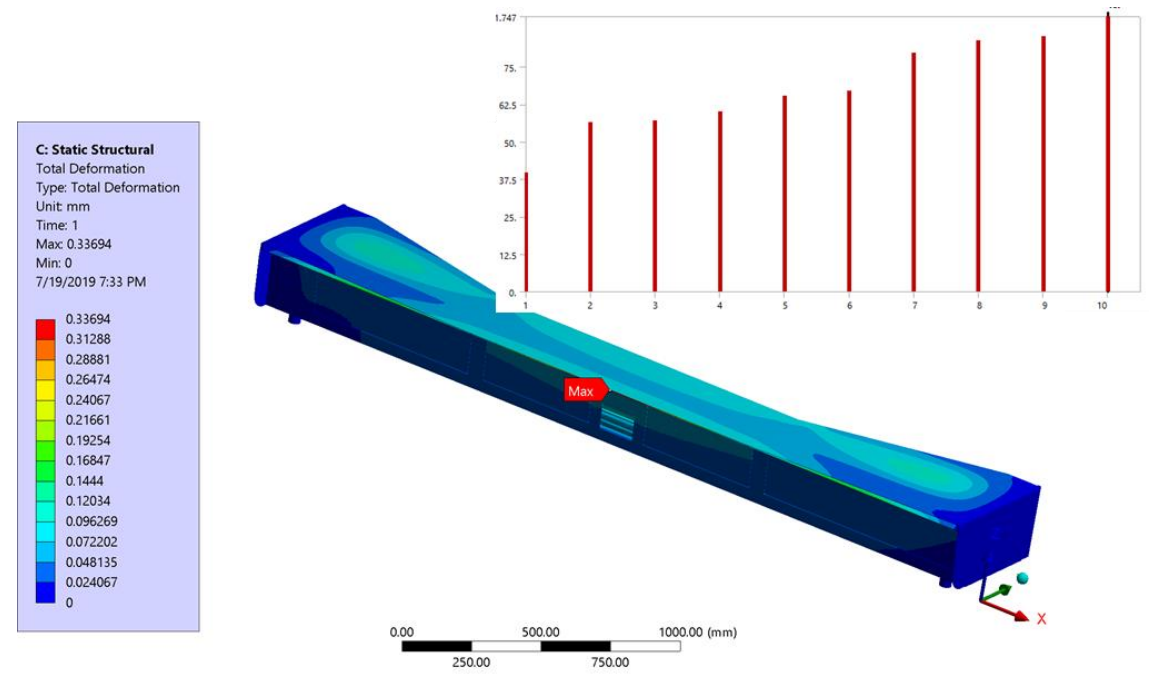

Figure 4

Deformed shape of integrated ODS housing for a static structural analysis and its natural frequencies in the range $0-100 \mathrm{~Hz}$

Finally, it is necessary to determine integrated ODS housing natural frequencies to ensure that they are not the resonance region, as the ODS housing would be exited form moving vehicle vibrations in noted frequency region and resonance would occur. The natural frequencies of integrated ODS housing with of all the internal components and added ballast were determined by modal analysis (with static structural pre-stress analysis) in ANSYS Workbench software. The modal analysis was performed for a range between 0 and $100 \mathrm{~Hz}$. The results of the modal analysis of a deformed ODS housing are shown in Figure 4. The primary natural 
frequency is close to $38 \mathrm{~Hz}$ which is well within the isolation range $(>10.5 \mathrm{~Hz})$ For the integrated ODS housing primary natural frequency the isolation efficiency of the designed vibration suppression system is $87.5 \%$.

\section{Experimental Determination of ODS Dynamic Response}

The evaluation of prototype of the freight onboard ODS, as well as the designed passive vibration isolation, was performed during the tests in operational traffic. The tests were performed on the railway line between Niš Marshaling Yard and station Ristovac, on the pan European 'corridor X'. A prototype of ODS was mounted on Locomotive 444-018, that pulled 21 wagons, with total mass of $1194 \mathrm{t}$ and a total train length of $458 \mathrm{~m}$, as shown on Figure 5. The test track length was $120 \mathrm{~km}$, the average speed was $34 \mathrm{~km} / \mathrm{h}$ and the test run lasted $3.5 \mathrm{~h}$ [22]. On the straight rail-tracks sections, between Niš Marshalling Yard and station Grdelica, the maximal train speed was $80 \mathrm{~km} / \mathrm{h}$.

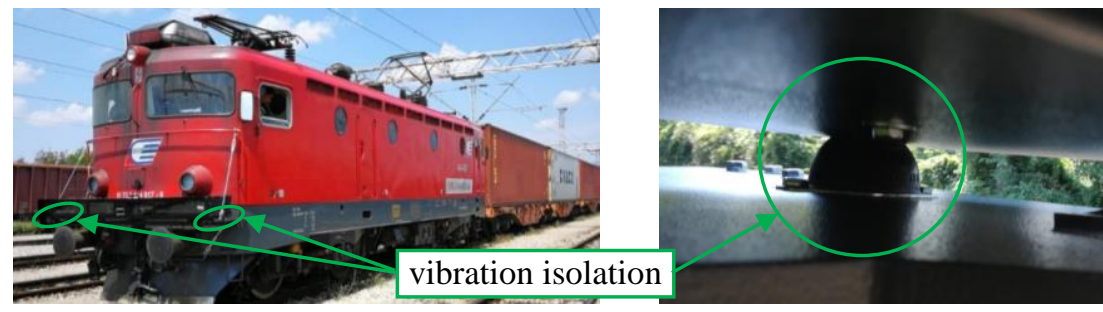

Figure 5

Locomotive 444-018 with mounted prototype ODS pulling a train during evaluation tests before leaving the Niš Marshalling Yard [22]

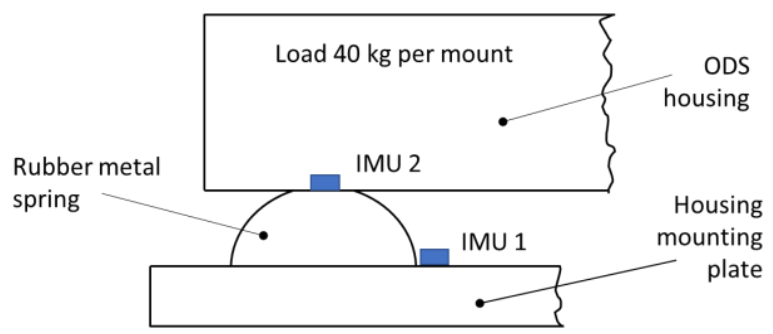

Figure 6

Positioning of IMU's on the housing mounting plate and inside the ODS housing

The vibration of the mounting plate and the ODS housing were recorded during the evaluation run by two triaxial Inertial Measurement Units (IMU's) positioned on the mounting plate and in the ODS housing as shown in Figure 6 . 
The acquisition device captured data from the IMU's at $75 \mathrm{~Hz}$. The vibrations were recorded on a straight line, in curves, during the crossing of the bridges and passing over the switches. As the train was following the operational speed profile, the vibrations were recorded at multiple train speeds.

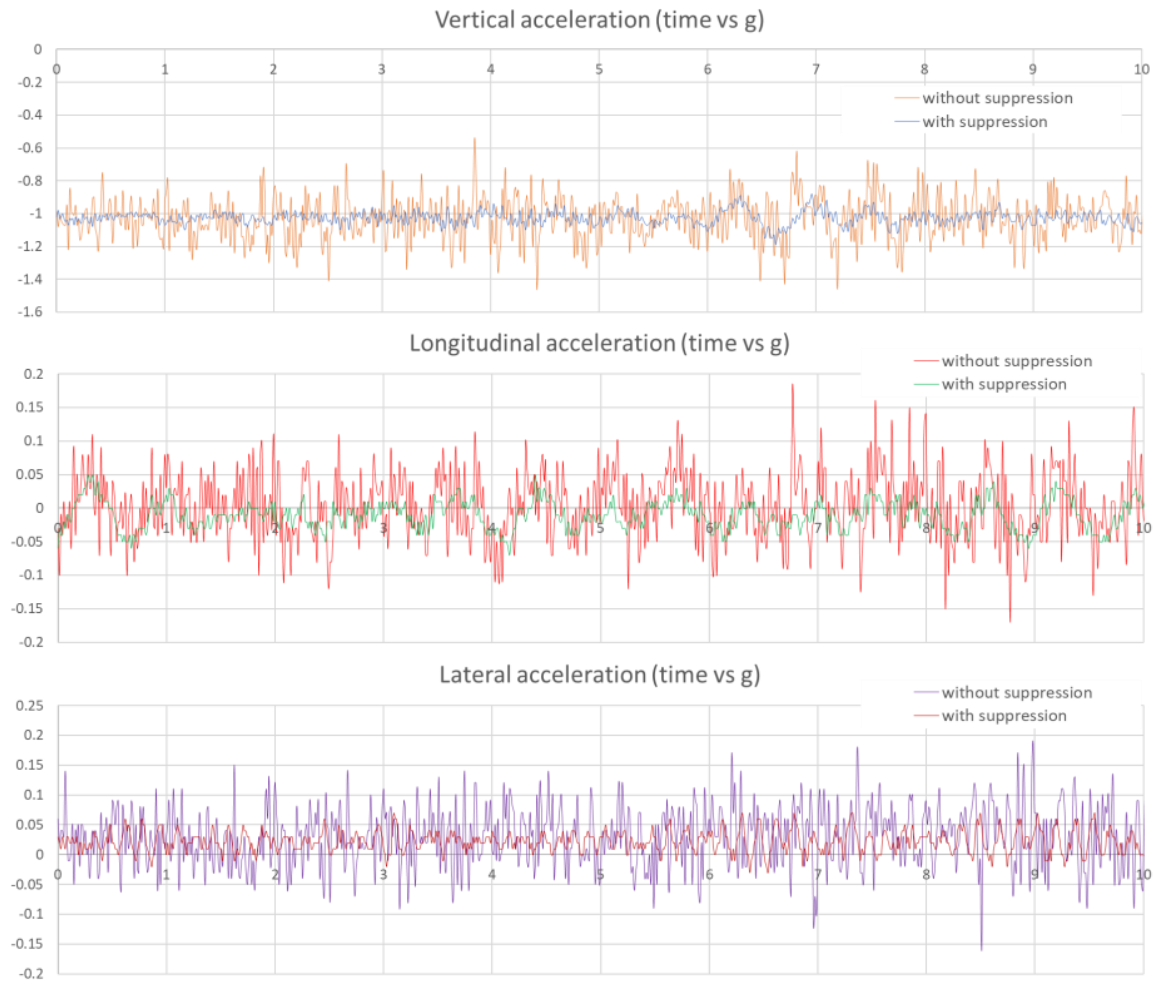

Figure 7

Vibrations of mounting plate (without suppression) and ODS housing (with suppression) on a straight part of the track at $80 \mathrm{~km} / \mathrm{h}$ in vertical, longitudinal and lateral direction

Figure 7 shows the 10 second interval of measured vibrations on a straight track at train speed of $80 \mathrm{~km} / \mathrm{h}$. It is evident from the diagrams shown on Figure 7 that the designed vibration isolation system significantly reduces the amplitude of vibrations in all three directions. As expected, due to largest deflection of the rubber - metal spring in the vertical direction, the degree of isolation is largest in the vertical direction. The vibration isolation performance in lateral direction is somewhat better than in longitudinal direction. This can be explained by lower excitation signal frequency in the longitudinal direction, which leads to higher transmissibility due to smaller value of ratio of excitation frequency and natural frequency of the vibrating system. 


\section{Prediction of Dynamic Response}

Following the experimental acceleration measurements in vertical direction from Figure 6 and mass per one mount of $40 \mathrm{~kg}$, the stochastic acting force in vertical direction without and with vibration suppression is calculated and presented in Figure 8 .

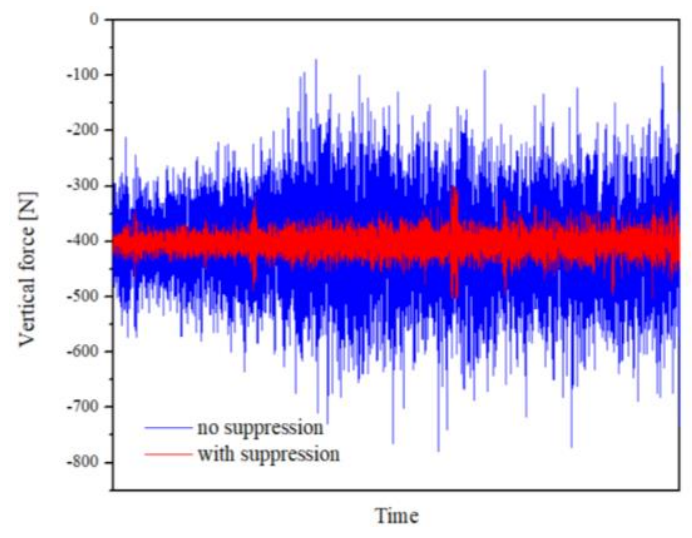

Figure 8

Calculated acting force in vertical direction

According to the designed vibration suppression used to isolate ODS housing from vibrations, the viscoelastic Voigt-Kelvin and Voigt-Maxwell models were applied for ODS housing stochastic vibrations analysis.

First, because of its simplicity, the Voigt-Kelvin model, was employed. This model consists of parallel connected spring and dashtop as shown in Figure 9.

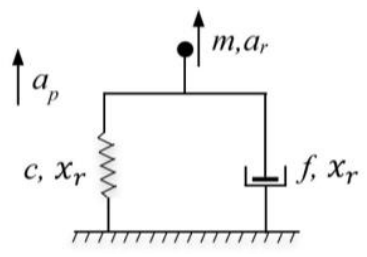

Figure 9

Voigt-Kelvin constitutive model

In applied Voigt-Kelvin constitutive model, $c$ is spring stiffness, $f$ is damping coefficient, $m$ presents the mass of analyzed system (load per ODS housing mount of $40 \mathrm{~kg})$ where $a_{r}\left(\ddot{x}_{r}\right)$ presents relative system acceleration which corresponds to measured acceleration data of ODS housing. On the other hand, $a_{p}$ is measured acceleration of the ODS housing mounting plate. According to dynamics of 
relative movement, the model from Figure 8 can be described with the following equation

$$
m \ddot{x}_{r}=-F_{c}(t)-F_{f}(t)+F_{p}^{i n}(t)
$$

The displacements in spring and dashtop elements are equal, $x_{c}=x_{f}=x_{r}$ where the acting forces in these elements are

$$
F_{c}(t)=c x_{r} \quad \text { and } \quad F_{f}(t)=f \dot{x}_{r}
$$

The expression $F_{p}^{i n}$ presents the inertial force according to acceleration $a_{\mathrm{p}}$

$$
F_{p}^{i n}=m \ddot{x}_{p}
$$

and it correspond to generated force given in Figure 8.

To obtain the accurate model which can be used to predict the dynamic response, it is necessary to determine the most suitable pair of parameters $c$ and $f$ for calculation of Voigt-Kelvin model which correspond to the experimental results for measured accelerations $\ddot{x}_{r}(t)$ and $\ddot{x}_{p}(t)$. For this purpose, the Monte Carlo simulation method is applied. According to equation (1) the appropriate model is given in Figure 10.

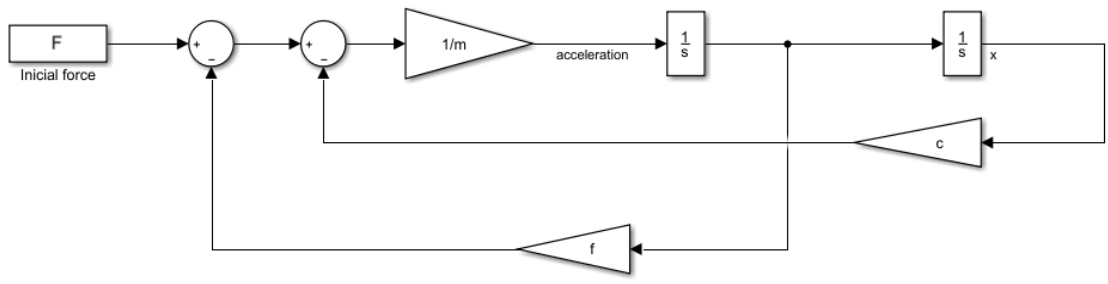

Figure 10

Simulation scheme for the system given by relation (1) and (2)

Following the Monte Carlo simulation method, the model from Figure 10 is simulated more 10000 times for various values of $c$ and $f$, where the starting (initial) values of this parameters were recalculated according to obtained elastic and viscoelastic parameters in [26]. These parameters are finally estimated for the model results which gives the smallest accelerations outputs error compared to experimental acceleration values for suppressed system. 


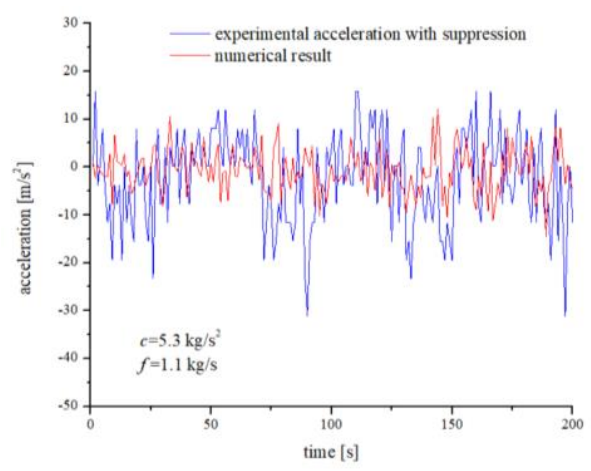

Figure 11

Voigt-Kelvin model acceleration output compared to experimental values in vertical direction

Figure 11 presents comparison of numerically determined acceleration values for a Voigt-Kelvin model and experimentally determined ODS housing response. It is evident from Figure 11 that Voigt-Kelvin model cannot accurately predict the ODS dynamic response. Although the acceleration direction is somewhat predicted, the amplitude values over time are largely missed. Because of its limitation on only two unknown parameters $c$ and $f$, the Voigt-Kelvin model application is limited to analysis of smaller displacements.

Therefore, it is necessary to use a more complex constitutive model, such as, Voigt model with two Maxwell elements marked with I and II in Figure 12. Contrary to Voigt-Kelvin model, this model is more suitable in modelling of larger disturbances.

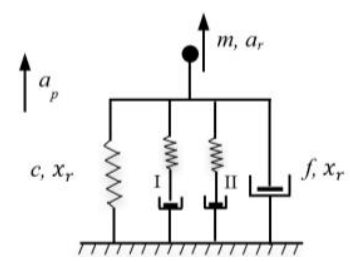

Figure 12

Voigt-Maxwell model

This applied Voigt-Maxwell constitutive model is described with following dynamic equations:

$m \ddot{x}_{r}=-F_{c}(t)-F_{f}(t)+F_{p}^{i n}(t)$

where $c_{1}, c_{2}$ are spring stiffness and $f_{1}, f_{2}$ are damping coefficient in Maxwell elements I and II and:

$F_{c}(t)=\left(c+c_{1}+c_{2}\right) x_{r}-c_{1} x_{1}-c_{2} x_{2} \quad$ and $\quad F_{f}(t)=f \dot{x}_{r}$ 
The relations between Maxwell elements are given as:

$$
\begin{aligned}
& f_{1} \dot{x}_{1}-c_{1} x_{r}+c_{1} x_{1}=0, \\
& f_{1} \dot{x}_{2}-c_{2} x_{r}+c_{2} x_{2}=0,
\end{aligned}
$$

where, $x_{1}$ and $x_{2}$ are movements between elements I and II respectively.

According to relations (4) and (6), the simulation scheme of applied VoigtMaxwell constitutive model is presented in Figure 13.

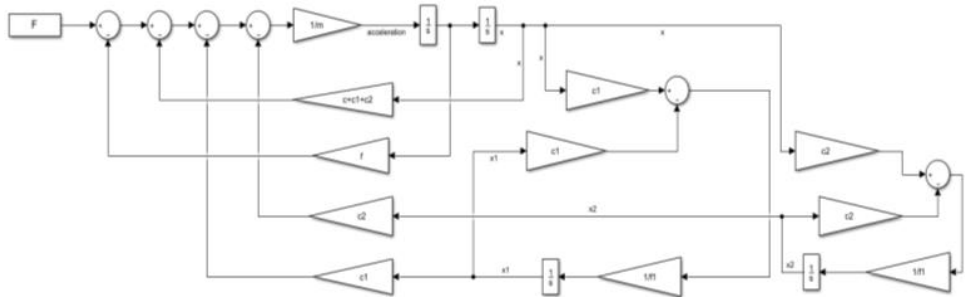

Figure 13

Simulation scheme of Voigt-Maxwell model

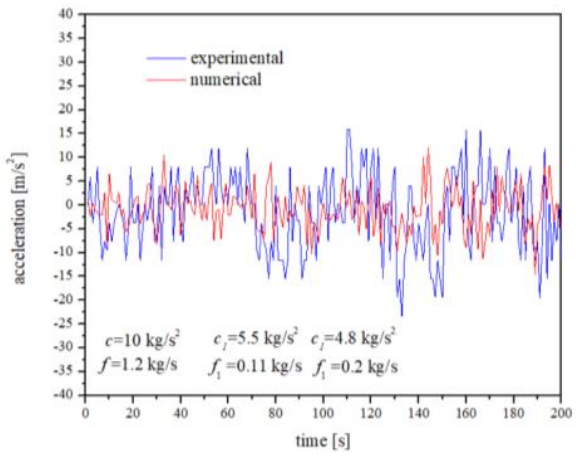

Figure 14

Voigt-Maxwell model acceleration output compared to experimental values in vertical direction

The spring stiffness $\left(c_{1}, c_{2}\right)$ and damping coefficients $\left(f_{1}, f_{2}\right)$ are determined by a same procedure as for Voigt-Kelvin model using the Monte Carlo simulation method.

Figure 14 presents comparison of numerically determined acceleration values for a Voigt-Maxwell constitutive model and experimentally determined ODS housing response. It is evident from Figure 14 that Voigt-Maxwell achieves better agreement between the numerically and experimentally determined acceleration values than Voigt-Kelvin model (Figure 11). There are still differences in prediction of acceleration directions and amplitude values, but the numerical and experimental determined acceleration amplitude values are somewhat comparable. 
According to upper and lower limits compared through time and using into account error in experimental measurements it can be concluded this model can be successfully used for further numerical system analysis and prediction. The addition of more parallel, connected Maxwell elements would increase the accuracy of prediction of dynamic response, but at expense of increased time for determination of constitutive model parameters.

\section{Conclusions}

As vibrations can distort the images acquired by vision-based sensors, it is necessary to isolate the sensors from the source of the vibrations. This paper presents the design of a passive vibration isolation system, for a freight train, by usage of bell-shaped rubber-metal springs. The design of the vibration isolation system was generated, based on measurements of the Serbia KARGO series 444 locomotive car body on a straight portion of the track at speed of $75 \mathrm{~km} / \mathrm{h}$. The presented system can isolate vibration with frequencies larger than $10.5 \mathrm{~Hz}$. The dynamic response of the ODS housing, as well as, the performance of the passive vibration isolation, was determined experimentally by measuring vibrations before and after the ODS vibration isolation system with the train in operational traffic. Based on experimental data, the designed vibration isolation system significantly reduces the amplitude of vibrations in all three directions. To predict the dynamic response of the vibration isolated ODS, viscoelastic VoigtKelvin and Voigt-Maxwell constitutive models were applied with real experimental data as system excitation. For both constitutive models the model parameters were determined by Monte Carlo simulation to achieve smallest error between predicted and experimental determined dynamic response. The comparison of results show that Voigt-Maxwell achieves better agreement between the numerically and experimentally determined acceleration values, over the Voigt-Kelvin model.

\section{Acknowledgement}

This research has been done in the framework of Horizon 2020 Shift2Rail project "Smart Automation of Rail Transport - SMART", grant ID 730836. The authors would like to thank the Serbian Railway Infrastructure for issuing permit and providing operational assistance for testing as well as Serbia KARGO for enabling the vibration measurement in operational environment.

\section{References}

[1] Kuchak, A. J. T., Marinkovic, D., Zehn, M., (2020) Finite element model updating-Case study of a rail damper. Structural Engineering and Mechanics, 73(1) pp. 27-35

[2] Carrella, A., Brennan, M. J., Waters, T. P., (2007) Static analysis of a passive vibration isolator with quasi-zero-stiffness characteristic. Journal of sound and vibration, 301(3-5) pp. 678-689 
[3] Kuchak, A. J. T., Marinkovic, D., Zehn, M., (2021) Parametric Investigation of a Rail Damper Design Based on a Lab-Scaled Model. Journal of Vibration Engineering \& Technologies, 9(1) pp. 51-60

[4] Li, L., Tan, L., Kong, L., Wang, D., Yang, H., (2018) The influence of flywheel micro vibration on space camera and vibration suppression. Mechanical Systems and Signal Processing, 100, pp. 360-370

[5] Zhao, G. W., Yuta, S. I., 1993, January. Obstacle detection by vision system for an autonomous vehicle. In 1993 Intelligent Vehicles Symposium, IV. Institute of Electrical and Electronics Engineers Inc. 1993, pp. 31-36

[6] Abolmaali, A., Fernandez, R., Kamangar, F., Ramirez, G., Le, T., (2008) Vibration Reduction and Control for Traffic Cameras: Technical Report (No. FHWA/TX-08/0-5251-2)

[7] SMART project, Deliverable 1.1. Obstacle Detection System Requirements and Specification

[8] Ristić-Durrant, D., Haseeb, M. A., Banić, M., Stamenković, D., Simonović, M., Nikolić, D., (2021) SMART on-board multi-sensor obstacle detection system for improvement of rail transport safety. Proceedings of the Institution of Mechanical Engineers, Part F: Journal of Rail and Rapid Transit, p. 09544097211032738

[9] Rysaeva, L. K., Korznikova, E. A., Murzaev, R. T., Abdullina, D. U., Kudreyko, A. A., Baimova, J. A., Lisovenko, D. S., Dmitriev, S. V., (2020) Elastic damper based on the carbon nanotube bundle. Facta Universitatis, Series: Mechanical Engineering, 18(1) pp. 001-012

[10] Banić, M., Stamenković, D., Miltenović, A., Jovanović, D., Tica, M., (2020) Procedure for the Selection of Rubber Compound in Rubber-Metal Springs for Vibration Isolation. Polymers, 12(8) p. 1737

[11] Sun, Y., Zhou, J., Thompson, D., Yuan, T., Gong, D., You, T., (2020) Design, analysis and experimental validation of high static and low dynamic stiffness mounts based on target force curves. International Journal of Non-Linear Mechanics, 126, p. 103559

[12] Cao, X., Wei, C., Liang, J., Wang, L., (2019) Design and dynamic analysis of metal rubber isolators between satellite and carrier rocket system. Mechanical Sciences, 10(1) pp. 71-78

[13] Zou, G. P., Liu, Z., Cheng, H. Z., Chang, Z. L., (2015) Effects of preloading and vibration level on the vibration characteristics of metal rubber damper. Journal of Vibration and Shock, 2015, p. 22

[14] Carrella, A., (2012) Nonlinear identifications using transmissibility: Dynamic characterisation of Anti Vibration Mounts (AVMs) with standard approach and nonlinear analysis. International Journal of Mechanical Sciences, 63(1) pp. 74-85 
[15] Ucar, H., Basdogan, I., (2018) Dynamic characterization and modeling of rubber shock absorbers: A comprehensive case study. Journal of Low Frequency Noise, Vibration and Active Control, 37(3) pp. 509-518

[16] Ramos, F. M. Vibration analysis of an engine mount. M.Sc. Thesis, Universidade Técnica de Lisboa Pais, Lisboa, Portugal, 2008: 1049-001

[17] Olsson, A. K. Finite Element Procedures in Modelling the Dynamic Properties of Rubber; Lund University: Lund, Sweden, 2007

[18] Pavlović, I. R., Pavlović, R., Janevski, G., Despenić, N., Pajković, V., (2020) Dynamic behavior of two elastically connected nanobeams under a white noise process. Facta Universitatis, Series: Mechanical Engineering, 18(2) pp. 219-227

[19] Németh, A., Fischer, S., (2021) Investigation of the Glued Insulated Rail Joints Applied to CWR Tracks. Facta Universitatis, Series: Mechanical Engineering

[20] Sysyn, M., Nabochenko, O., Kovalchuk, V., Przybyłowicz, M., Fischer, S., (2021) Investigation of interlocking effect of crushed stone ballast during dynamic loading. Reports in Mechanical Engineering, 2(1) pp. 65-76

[21] Smart Automation of Rail Transport - SMART, https://cordis.europa.eu/ project/id/730836

[22] SMART project, Deliverable 7.1. Report on evaluation of developed SMART technologies

[23] SMART project, Deliverable 2.2.- Design of the passive vibration isolation system

[24] Trelleborg IAVS Catalogue, 2017

[25] Banić, M., Stamenković, D., Miltenović, A., Simonović, M., Milošević, M., (2019) Design of Housing and Vibration Suppression for Obstacle Detection System in Railways. Proceedings of $24^{\text {th }}$ International conference "Current Problems in Rail Vehicles" - PRORAIL 2019, 1, Žilina, Slovakia, 17-19 September, pp. 23-31

[26] Pavlović I., Ćirić I., Đekić P., Nikolić V., Pavlović R., Ćojbašić Ž., Radenković G. (2013) Rheological model optimization using advanced evolutionary computation for the analysis of the influence of recycled rubber on rubber blend dynamical behavior, Meccanica, 48:2467-2477 